ETHNIC DISPARITIES IN CANCER INCIDENCE AND SURVIVAL AMONG THE OLDEST OLD IN THE UNITED STATES

J.L. Krok-Schoen ${ }^{3,2}$, R.D. Baltic ${ }^{2}$, J.L. Fisher ${ }^{1}, 1$. Arthur

G. James Cancer Hospital and Richard J. Solove Research

Institute, Columbus, Ohio, 2. Comprehensive Cancer

Center, The Ohio State Univer, Columbus, Ohio, 3. School

of Health and Rehabilitation Sciences, College of Medicine,

The Ohio State University, Columbus, Obio

Two of the fastest growing segments of the U.S. population are the oldest old, people aged $\geq 85$ years, and Hispanics. Currently, no studies have examined ethnic disparities in cancer incidence and survival in the $\geq 85$ population. This study sought to examine potential ethnic disparities in cancer incidence and survival rates among the oldest old using data from the SEER Program. Cases diagnosed with one of the leading four cancer sites (lung and bronchus, colon and rectum, female breast, prostate) were reported to one of 18 SEER registries. Differences in cancer incidence were examined for cases aged $\geq 85$ years and diagnosed 1992-2013. Five-year relative cancer survival probability was examined for Hispanic and non-Hispanic adults aged $\geq 85$ years diagnosed 2006-2012. Results indicated that from 1992-2013, among those aged $\geq 85$ years, non-Hispanics had higher incidence compared to Hispanics for colon and rectum, lung and bronchus, female breast, and prostate cancers. Five-year survival probability for cancers of all stages combined was higher for non-Hispanics than Hispanics in this age group. However, Hispanics had higher survival probability of colorectal cancer diagnosed at regional $(67.2 \%$ vs. $60.5 \%)$ and distant $(5.4 \%$ vs. $3.8 \%)$ stages than non-Hispanics, respectively. Hispanics also had higher survival probability of lung and bronchus cancer diagnosed at regional $(15.9 \%$ vs. $12.7 \%)$ and distant $(2.8 \%$ vs. $2.2 \%)$ stages than non-Hispanics, respectively. In summary, ethnic differences in cancer incidence and survival probability exist within the $\geq 85$ population. Continued efforts are needed to understand and reduce ethnic disparities in cancer prevention and treatment among this population.

\section{A MIXED METHODS APPROACH TO EXAMINING POSTTRAUMATIC GROWTH IN OLDER CANCER SURVIVORS}

A.I. Hoogland, Moffitt Cancer Center, Tampa, Florida

A wealth of research has been conducted on cancer survivorship and posttraumatic growth (PTG; defined as positive change following the struggle associated with a trauma), but little research has investigated PTG in older samples. This lack of scholarship is surprising, considering cancer diagnoses are especially prevalent in older adults. To address this gap in our knowledgebase, a population-based random sample of older cancer survivors $(N=56)$ was recruited through the Kentucky Cancer Registry. Participants mailed in questionnaires that included several validated surveys, including the Posttraumatic Growth Inventory (PTGI). Overall, participants reported high levels of posttraumatic growth (PTG Mean $=54.85 ; S D=28.31$ ), which was positively associated with personal significance of the cancer diagnosis $(p=.04)$. Normed PTGI Subscale scores were nuanced such that participants felt more appreciation of life, but a decreased sense of having new possibilities. Even among older adults, there were age trends such that "older" older adults reported less PTG than "younger" older adults $(p=.002)$, and were less personally impacted by a cancer diagnosis $(p<.001)$. Qualitatively, a reliance on faith and a sense of acceptance emerged as themes, consistent with previous literature identifying a shift in perspective with age that allows for embracing changes in health status. It is feasible that older adults are better able to accept traumatic illnesses as a natural part of the life course, as has been seen in other studies on late-life illness.

\section{SESSION 785 (POSTER)}

\section{CAREGIVING II}

\section{INFORMAL CAREGIVER BURDENS AND THE SUBSEQUENT COGNITIVE STATUS OF THE CARE RECIPIENT}

D. Lloyd, M.P. Aranda, School of Social Work, University of Southern California, Los Angeles, California

Studying factors influencing caregiver burden implies that, in addition to the caregivers' quality of life, such burden may influence the health trajectory of care recipients. Five domains of caregiver burden are analyzed in relation to care recipients' change in cognitive status one year later using National Health and Aging Trends Study and linked National Study on Caregiving data. A sample of 703 care recipients whose baseline cognitive status is not probable dementia, and their primary informal caregivers, are included. Caregiver burden dimensions of social limitation, role overload, and psychological distress are associated with care recipients' transition to probable dementia, while positive mood and financial burden are not, in separate regression models with controls for care recipients' sex, age, race/ethnicity, marital status, education, self-rated health, and "possible dementia" status. When all five domains are included in the same model, social limitation is the sole net predictor of cognitive status change. Determinants of caregiver burden are examined as well. Simultaneous regression of all five burden measures on care recipient and caregiver characteristics reveals several factors. Notable predictors of specific caregiver burdens are recipients' relationship to the caregiver, their health, and caregivers' employment status, marital status, and gender. Caregiver commitment to a set schedule of caregiving is associated with social limitation, role overload, and psychological distress. Distress is greater among sons who are primary caregivers, compared with those who are the care recipients' spouse, while being married (but not the recipients' spouse), is protective. Additional findings will be reported, and their implications discussed.

\section{PARTICIPATORY ARTS IN THE HOME TO SUPPORT WELLNESS IN DEMENTIA CAREGIVER AND CARE RECIPIENT DYADS}

R. Poulos ${ }^{2}$, D. Harkin ${ }^{1}$, E.R. Beattie ${ }^{3}$, C. Cunningham ${ }^{1,4}$, C. Poulos ${ }^{1,2}$, 1. HammondCare, Sydney, New South Wales, Australia, 2. University of New South Wales, Sydney, New South Wales, Australia, 3. Queensland University of Technology, Brisbane, Queensland, Australia, 4. University of Edinburgh, Edinburgh, Scotland, United Kingdom 